\title{
Qualidade do amendoim armazenado em diferentes embalagens
}

\author{
Quality of peanuts stored in different packaging \\ Calidad del maní almacenado en diferentes envases
}

\section{Resumo}

O amendoim (Arachis hypogaea L.) armazenado em ambiente não controlado sofre constantes interações, espontânea e inevitável, diretamente influenciadas pela temperatura e umidade relativa, cujas transformações, no qual ocorre a deterioração dos lipídios e constitui-se em um dos problemas técnicos mais importantes na indústria de alimentos. Dependendo das condições ambientais, a embalagem de baixa permeabilidade ao vapor de água para armazenar o produto apresenta grande importância nas etapas pós-colheita, conferindo proteção e minimizando as interações entre produto-ambiente. $\mathrm{O}$ estudo teve como objetivo analisar o impacto de diferentes materiais para embalagem no armazenamento do amendoim. O experimento comparando as embalagens foi realizado em esquema fatorial $2 \times 4$, sendo dois tipos de embalagem (ráfia e juta) e quatro períodos de armazenamento (60, 120, 220 e 290 dias), com quatro repetições. $\mathrm{O}$ amendoim foi armazenado na forma de vagens completas e as amostras mantidas sobre condições de ambiente não controladas. Avaliou-se os teores de água da casca e da semente, a condutividade elétrica e o índice de velocidade de germinação das sementes. Os dados foram submetidos a análise de variância e as médias comparadas pelo teste Tukey, com 5\% de significância. $\mathrm{O}$ acondicionamento em embalagens de juta proporcionou melhores condições de armazenamento em relação a embalagem de ráfia. A temperatura e umidade relativa afetam na umidade de equilíbrio das sementes de amendoim armazenadas.

Palavras-chave: Arachis hypogaea L.; Oleaginosas; Pós-colheita; Sorção.

\begin{abstract}
Peanuts (Arachis hypogaea L.) stored in an uncontrolled environment undergo constant interactions, spontaneous and inevitable, directly influenced by temperature and relative humidity, whose transformations occur the deterioration of lipids and constitutes one of the most important technical problems in the food industry. Depending on environmental conditions, the packaging of low permeability to water vapor to store the product is of great importance in the postharvest stages, providing protection and minizing the interactions between product-environment. The study aimed to analyze the impact of different packaging materials on peanut storage. The experiment comparing the packages was carried out in a $2 \times 4$ factorial scheme, being two types of packaging (raphy and jute) and four storage periods $(60,120$, 220 and 290 days), with four replications. Peanuts were stored in the form of complete pods and samples were kept under uncontrolled ambient conditions. The water contents of the bark and seed, the electrical conductivity and the germination speed index of the seeds were evaluated. The data were submitted to variance analysis and the means compared by the Tukey test, with $5 \%$ significance. The packaging in jute packaging provided better storage
\end{abstract}


conditions in relation to the packaging of atrophy. The temperature and relative humidity affect the equilibrium humidity of stored peanut seeds.

Keywords: Arachis hypogaea, L; Oilseeds; Post-harvest; Sorption.

\begin{abstract}
Resumen
El maní (Arachis hypogaea L.) almacenado en ambiente incontrolado sufre interacciones constantes, espontáneas e inevitables, directamente influenciadas por la temperatura y la humedad relativa, cuyas transformaciones, en las que se produce el deterioro de los lípidos, constituye uno de los problemas técnicos más importantes de la industria alimentaria. Dependiendo de las condiciones ambientales, el embalaje con baja permeabilidad al vapor de agua para el almacenamiento del producto es de gran importancia en las etapas pos cosecha, brindando protección y minimizando las interacciones entre producto y ambiente. El estudio tuvo como objetivo analizar el impacto de diferentes materiales de embalaje en el almacenamiento de maní. El experimento de comparación de paquetes se realizó en esquema factorial $2 \times 4$, con dos tipos de empaque (rafia y yute) y cuatro períodos de almacenamiento $(60,120,220$ y 290 días), con cuatro repeticiones. Los cacahuetes se almacenaron como vainas completas y las muestras se mantuvieron en condiciones ambientales incontroladas. Se evaluó el contenido de agua de la cáscara y la semilla, la conductividad eléctrica y el índice de velocidad de germinación de las semillas. Los datos se sometieron a análisis de varianza y se compararon las medias mediante la prueba de Tukey, con una significancia del 5\%. El embalaje en embalaje de yute proporcionó mejores condiciones de almacenamiento en comparación con el embalaje de rafia. La temperatura y la humedad relativa afectan la humedad de equilibrio de las semillas de maní almacenadas.
\end{abstract}

Palabras clave: Arachis hypogaea L.; Oleaginosas; Pos cosecha; Sorción.

\title{
1. Introdução
}

Devido suas propriedades higroscópicas, o amendoim (Arachis hypogaea L.) é capaz de ceder ou absorver água do ambiente de acordo com a pressão de vapor de água da atmosfera do ambiente ao qual está exposto tendendo ao equilíbrio higroscópico. Dito isto, as condições do ambiente durante o período de armazenamento são de suma importância para a conservação da qualidade fisiológica do grão, pois este está em constante interação com o ar ambiente.

Grãos armazenados continuam com processos metabólicos ativos mantendo sua taxa respiratória reduzida, mas podendo ser influenciada conforme as condições de temperatura e umidade relativa do ar acelerando a deterioração através de reações bioquímicas, fisiológicas ou metabólicas (Nunes et al., 2021). Condições de baixa temperatura e umidade retardam o processo de deterioração do produto favorecendo sua conservação (Jaques et al., 2018).

A instabilidade química dos lipídeos constituintes afeta a qualidade final do produto devido este manter a atividade respiratório mesmo armazenado (Oliveira et al., 2020). No período de armazenamento a escolha da embalagem para acondicionamento do produto é de grande importância por este conferir proteção contra possíveis ataques de insetos, presença de patógenos e às constantes interações com o ar atmosférico.

A embalagem utilizada é um dos fatores responsáveis pelas taxas de trocas de vapor de água entre o produto armazenado e o ambiente, que necessita de temperatura e umidade relativa ideais para manter a integridade e qualidade, podendo ser de material permeável, semipermeável ou impermeável (Smaniotto et al., 2020). Embalagens com maior hermeticidade proporcionam melhores condições para a conservação de grãos por manter constante o seu teor de água durante o armazenamento reduzindo as trocas de água com o ambiente (Amaral et al., 2019).

$\mathrm{O}$ trabalho teve como objetivo analisar o efeito das condições de armazenamento sobre as características das sementes de amendoim, considerando diferentes materiais de armazenagem.

\section{Metodologia}

O estudo foi desenvolvido no laboratório de plantas medicinais e tecnologia pós-colheita pertencente a Universidade Estadual de Maringá (UEM), localizado no campus sede em Maringá-PR. O material utilizado no estudo, amendoim em vagem, foi obtido com produtor da agricultura familiar, no município de Campo Mourão-PR, apresentando como características físicas peso específico aparente de $0,547 \mathrm{Mg} \mathrm{m}^{-3}$, peso especifico real de 1,076 $\mathrm{Mg} \mathrm{m}^{-3}$, porosidade de 49,09\% e 
teor de água de $4,72 \%$ (bs).

$\mathrm{O}$ experimento foi desenvolvido em delineamento inteiramente casualizado em esquema fatorial $2 \times 4$, sendo dois tipos de embalagem para armazenamento (ráfia e juta) e quatro períodos de armazenamento (56, 120, 220 e 290 dias), com quatro repetições. As embalagens de ráfia e juta, com capacidade aproximada de $100 \mathrm{~g}$ por amostra, foram mantidas sobre condições não controladas de temperatura e umidade relativa.

Para determinação do teor de água na casca e na semente, foi utilizado o método gravimétrico com estufa de circulação forçada de ar à $105^{\circ} \mathrm{C}$ durante 24 horas. Para determinação da condutividade elétrica, foram separados aleatoriamente 25 sementes, após pesagem, colocadas em copo de polietileno, capacidade de $75 \mathrm{~mL}$, adicionando $50 \mathrm{~mL}$ de água destilada e mantidas em câmara BOD (Demanda Química do Oxigênio) por 24 horas. Posteriormente realizou-se a leitura da CE em solução com condutivímetro digital. No teste de germinação, 50 sementes foram colocadas em rolo de papel, sendo mantido em câmara de germinação à $25^{\circ} \mathrm{C}$, com contagem do número de sementes germinadas aos 10 dias (Brasil, 2009), com quatro repetições por tratamento.

Foi realizada análise exploratória dos dados, com determinação da correlação entre variáveis analisadas. Os dados foram submetidos a análise de variância, e as médias comparadas pelo teste Tukey com significância de 5\% utilizando o software Sisvar (Ferreira, 2019).

\section{Resultados e Discussão}

Considerando que o amendoim apresenta, em composição centesimal, elevado teor de lipídeos (Rodrigues et al., 2016), o impacto de falhas no armazenamento pode ser mais elevado sobre características físicas e sobre o potencial fisiológico. Os dados revelaram que o tempo de armazenamento apresentou efeito significativo $(\mathrm{p}<0,05)$ para o potencial germinativo e para condutividade elétrica (Figura 1). Durante o armazenamento, independente da embalagem, houve uma redução acentuada no percentual de sementes germinadas, sendo após 100 dias inferior a 70\%.

A semente apresenta máximo potencial germinativo na maturação fisiológica, com decréscimo no decorrer do tempo (Jesus et al., 2020). A redução do potencial de germinação se relacionada, entre os fatores envolvidos, com as condições de temperatura e umidade do ambiente que pela ausência de controle promovam condição para constante variação das propriedades higroscópicas do produto, proporcionando fenômenos de sorção e adsorção (Sarath et al., 2016; Costa et al., 2014), favorecendo a atividade metabólica e prejudicando a conservação (Erten \& Cadwallader, 2017).

Figura 1. Influência do período de armazenamento nas características de sementes de amendoim armazenadas em ráfia e juta: A) na germinação; e B) condutividade elétrica.
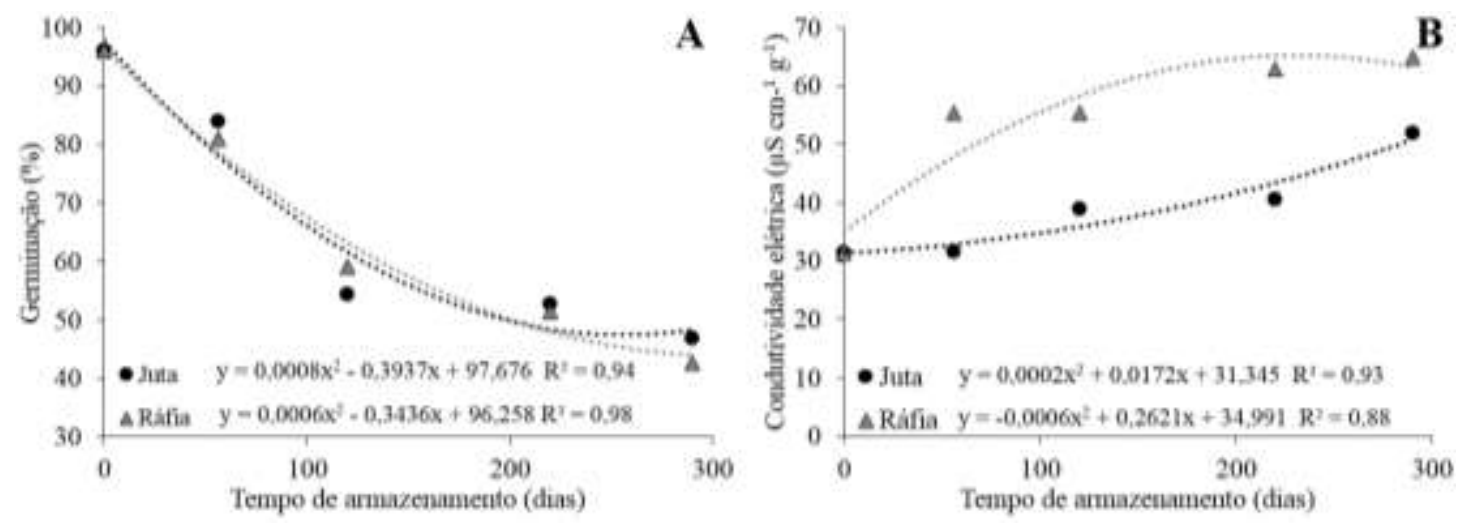

Fonte: Autores. 
Nas sementes armazenadas, as reações bioquímicas a nível celular podem gerar perdas de caráter qualitativo e quantitativo. $\mathrm{O}$ ataque de pragas, desenvolvimento de fungos e a atividade respiratória do produto aceleram a velocidade das reações e o potencial do processo deteriorativo (Nunes et al., 2021). No período de armazenamento, a embalagem pode atuar diretamente como um obstáculo no processo, reduzindo as trocas entre o produto e o ambiente, principalmente de materiais permeáveis e assim manter a qualidade comercial por longos períodos (Amaro et al., 2019; Smaniotto et al., 2020). Entretanto, nas condições de acondicionamento adotadas, as embalagens (ráfia e juta) permitiram a troca facilitada de vapor d'água e temperatura do produto com o ambiente. Ao considerar o impacto das embalagens sobre o armazenamento do amendoim, foi constatado efeito significativo apenas para os índices de condutividade elétrica das sementes (Tabela 1).

Tabela 1. Valores médios de germinação, teor de água da casca e da semente, e condutividade elétrica de sementes de amendoim armazenadas em embalagem de ráfia e juta em ambiente não controlado.

\begin{tabular}{cccccccc}
\hline \multirow{2}{*}{ Variável } & \multirow{2}{*}{ Embalagem } & \multicolumn{5}{c}{ Tempo de armazenamento (dias) } & CV \\
\cline { 3 - 6 } & & 0 & 56 & 120 & 220 & 290 & $(\%)$ \\
\hline \multirow{2}{*}{ Germinação (\%) } & Juta & $96^{\text {ns }}$ & 84 & 54,40 & 52,8 & 46,85 & \multirow{2}{*}{15,18} \\
& Ráfia & 96 & 81 & 59,11 & 51,42 & 42,66 & \\
\hline \multirow{2}{*}{ Teor de água da casca (\% bs) } & Juta & $5,47^{\text {ns }}$ & 6,93 & 8,33 & 8,66 & 11,22 & \multirow{2}{*}{25,4} \\
& Ráfia & 5,47 & 10,97 & 8,43 & 6,72 & 9,00 & \\
\hline \multirow{2}{*}{ Teor de água da semente $(\%$} & Juta & $4,72^{\text {ns }}$ & 5,56 & 6,51 & 5,76 & 5,45 & \multirow{2}{*}{22,64} \\
bs) & Ráfia & 4,72 & 5,03 & 5,82 & 4,47 & 5,82 & \\
\hline Condutividade elétrica & Juta & $31,33 \mathrm{a}$ & $31,56 \mathrm{a}$ & $38,82 \mathrm{a}$ & $40,58 \mathrm{a}$ & $51,87 \mathrm{a}$ & \multirow{2}{*}{44,01} \\
\hline$\left(\mu \mathrm{S} \mathrm{cm}^{-1} \mathrm{~g}^{-1}\right)$ & Ráfia & $31,33 \mathrm{a}$ & $55,29 \mathrm{~b}$ & $55,29 \mathrm{~b}$ & $62,8 \mathrm{~b}$ & $64,82 \mathrm{~b}$ & \\
\hline
\end{tabular}

*Letras diferentes na coluna, para cada variável analisada, diferem entre si pelo teste Tukey $(\mathrm{p} \leq 0,05)$.

Fonte: Autores.

Em relação ao teor de água do fruto do amendoim, comparado à semente, a casca apresenta teores de água mais elevados (Tabela 1), sugerindo estar desempenhando uma função que atua como barreira física contra danos físicos e variações do ambiente (Oliveira et al., 2020). Embora observada uma variação em números absolutos no teor de água das sementes e esperada pelas alterações da condição do ambiente no decorrer do armazenamento, esta não foi significativa (Tabela 1).

Para fins comerciais um fator importante no armazenamento de sementes refere-se as condições do seu acondicionamento, onde o ambiente refrigerado ameniza a atividade respiratória (Amaro et al., 2019). Considerando a importância das sementes no processo produtivo, os índices de qualidade e sanidade inicial devem ser considerados para o armazenamento do lote (Sarto, 2019; Manandhar, Milindi \& Shah, 2018).

A escolha do material da embalagem para o acondicionamento do produto pode ser uma medida para minimizar as perdas causadas por insetos (Silva et al., 2021), no período do armazenamento, garantindo a qualidade do produto no momento da comercialização (Silva Neto \& Santos, 2019). Pois, embalagens inadequadas e condições desfavoráveis durante o armazenamento refletem em prejuízos quantitativos e qualitativos no setor agroindustrial.

O manejo do produto em campo, na colheita e em processos pós-colheita, como secagem, aliado a presença de danos, oriundos do ataque de pragas e fungos, pode afetar a integridade da estrutura de sementes, provocando perdas de eletrólitos e proporcionando o desenvolvimento de patógenos (Saath et al., 2010; Ferreira et al., 2017). A integridade da membrana pode ser mensurada pela determinação de condutividade elétrica, que reflete a presença de íons em solução (Saath et al., 2017; Wenneck et al., 2020).

Nas condições do estudo, os índices da condutividade elétrica foram influenciados pelo tempo de armazenamento 
(Figura 1B) e pela embalagem de acondicionamento (Tabela 1). No armazenamento há tendência de redução na qualidade do produto, podendo ser verificada através da condutividade elétrica, com grau de quebra de qualidade e velocidade sendo influenciado principalmente por condições de alta temperatura e umidade relativa elevada (Jaques et al., 2018; Ely, 2018; Ziegler et al., 2017). Conforme Figura 1B, estima-se que a condutividade elétrica apresente incremento de $0,26 \mu \mathrm{Scm}^{-1} \mathrm{~g}^{-1}$ para o amendoim armazenado em ráfia e $0,2 \mu \mathrm{S} \mathrm{cm}^{-1} \mathrm{~g}^{-1}$ para amendoim armazenado em juta, a cada dia de armazenamento.

No decorrer do tempo, o armazenamento do amendoim em ráfia se apresentou menos promissor, considerando os valores superiores para os índices de condutividade elétrica (Tabela 1). Embora ambos os materiais (juta e ráfia) apresentem permeabilidade à água e troca de gases e, que a estrutura da embalagem de ráfia sugere ser uma barreira para entrada da insetos, quando há presença de focos de pragas não detectadas no início do armazenamento, a barreira atuou como limitante a movimentação entre frutos, elevando o dano pontual. Nesse sentido, a presença de pragas pode estar entre os fatores que diferiram em termos os índices de condutividade elétrica para o tipo de embalagens, considerando que não houve medidas de controle fitossanitário no recebimento do produto.

Tabela 2. Correlação das variáveis de sementes de amendoim acondicionadas em embalagem de ráfia e juta armazenadas em ambiente não controlado durante o período de 290 dias.

\begin{tabular}{llllllllll}
\hline & Tempo & G Juta & G Ráfia & UC Juta & UC Ráfia & US Juta & US Ráfia & CE Juta & CE Ráfia \\
\hline Tempo & 1,00 & - & - & - & - & - & - & - & - \\
G Juta & $-0,91$ & 1,00 & - & - & - & - & - & - & - \\
G Ráfia & $-0,96$ & 0,99 & 1,00 & - & - & - & - & - & - \\
UC Juta & 0,96 & $-0,91$ & $-0,95$ & 1,00 & - & - & - & - & - \\
UC Ráfia & 0,15 & $-0,16$ & $-0,21$ & 0,31 & 1,00 & - & - & - & - \\
US Juta & 0,32 & $-0,66$ & $-0,57$ & 0,40 & 0,39 & 1,00 & - & - & - \\
US Ráfia & 0,35 & $-0,49$ & $-0,46$ & 0,59 & 0,48 & 0,49 & 1,00 & - & - \\
CE Juta & 0,95 & $-0,86$ & $-0,90$ & 0,97 & 0,11 & 0,24 & 0,56 & 1,00 & - \\
CE Ráfia & 0,85 & $-0,86$ & $-0,90$ & 0,85 & 0,54 & 0,61 & 0,35 & 0,71 & 1,00 \\
\hline
\end{tabular}

*G: Porcentagem de Germinação; UC: Umidade da casca; US: Umidade da semente; CE: Condutividade Elétrica. Fonte: Autores.

Como medida indireta de integridade celular e reflexo de reações bioquímicas e enzimáticas (Ziegler et al., 2017), o índice da condutividade elétrica apresenta correlação com a germinação das sementes de amendoim, cujo valor foi de $-0,86$ e 0,9 para juta e ráfia, respectivamente (Tabela 2).

No armazenamento de amendoim, os fatores envolvidos na colheita e pós-colheita podem ainda influenciar a presença de microrganismos que produzem aflotoxinas, que comprometem a qualidade comercial do produto (Saath et al., 2021). Considerando que no decorrer do tempo de armazenamento há uma redução, espontânea e inevitável, em características qualitativas do produto, os processos e fatores envolvidos devem ser identificados e acompanhados para minimizar a intensidade de danos.

\section{Considerações Finais}

O acondicionamento do amendoim em embalagens de juta propiciou melhores condições para a conservação do produto armazenado comparado à embalagem de ráfia;

O tempo de armazenamento influencia diretamente no potencial germinativo da semente, independente da embalagem adotada para o armazenamento do amendoim. 
Novos estudos envolvendo as condições do ambiente de armazenamento são necessários para a compreensão das alterações nos grãos no decorrer do tempo, sendo fundamental para o desenvolvimento de técnicas e manejo visando reduzir perdas pós-colheita.

\section{Agradecimentos}

O presente trabalho foi realizado com apoio da Coordenação de Aperfeiçoamento de Pessoal de Nível Superior Brasil (CAPES) - Código de Financiamento 001.

\section{Referências}

Amaral, J. R. S., Peixoto, T., Pires, E. K. D., Lacerda, R. B., Cirino, K. F. S., Milan, M. D., \& Ferreira, R. B. (2019). Efeito do ambiente e da embalagem no teor de água de grãos de arroz armazenados. Revista Biodiversidade, 18 (3), 80-88.

Amaro, H. T. R., Araujo, E. F., Araujo, R. F., Dias, L. A. S., David, A. M. S. S., \& Silva, F. W. S. (2019). Secagem e armazenamento de sementes de culturas oleaginosas. Pesquisa Agropecuária Gaúcha, 25 (n.1/2), 105-119.

Corrêa, P. C., Goneli, A. L. D., Resende, O., \& Ribeiro, D. M. (2007). Obtenção e modelagem das isotermas de dessorção e do calor isotérico de dessorção para grãos de trigo. Revista Brasileira de Produtos Agroindustriais, 7, 39-48.

Costa, L. M., Resende, O., \& Oliveira, D. E. C. (2014). Determinação das isotermas de equilíbrio higroscópico de frutos de crambe pelo método dinâmico. Bioscience Journal, 31 (2), 382-391.

Ely, A. (2018). Redução da qualidade de grãos de soja durante o armazenamento em diferentes condições de umidade e temperatura. Revista Thema, 15 (2), 506-520. https://doi.org/10.15536/thema.15.2018.506-520.859

Erten, E. S., \& Cadwallader, K. R. (2017). Identification of prediminant aroma componentes of raw, dry roasted and oil roasted almonds. Food Chemistry, 217, 244-253.

Ferreira, D. F. (2019). SISVAR: a computer analysis system to fixed effects Split plot type designs. Revista Brasileira de Biometria, 37 (4), $529-535$.

Ferreira, L. B. S., Fernandes, N. A., Aquino, L. C., Silva, A. R., Nascimento, W. M., \& Leão-Araújo, E. F. (2017). Temperature and seed moisture content affect electrical conductivity test in pea seeds. Journal of Seed Science, 39 (4), 410-416.

Jaques, L. B. A., Ely, A., Hareberlin, L., Medeiros, E. P., \& Paraginski, R. T. (2018). Efeitos da temperatura e da umidade dos grãos de milho nos parâmetros de qualidade tecnológica. Revista Eletrônica Científica Da UERGS, 4 (3), 409-420. https://doi.org/10.21674/2448-0479.43.409-420

Jesus, M. A., Barbosa, R. M., \& Vieira, R. D. (2020). Controle estatístico de processo aplicado ao processamento de sementes de amendoim. South American Sciences, 1 (2), 1-13.

Manandhar, A., Milindi, P., \& Shah, A. (2018). An Overview of the Post-Harvest Grain Storage Practices of Smallholder Farmers in Developing Countries. Agriculture, 8 (4), 1-21.

Ministério da Agricultura do Abastecimento e da Reforma Agrária (2009). Regras para análise de Sementes: Secretaria Nacional de Defesa Agropecuária. 399 p.

Mohapatra, D., \& Rao, P. S. (2005). A thin layer drying model of parboiled wheat. Journal of Food Engineering, 66 (4), $513-518$.

Nunes, C. F., Medeiros, E. P., Haeberlin, L., Bihalva, N., \& Paraginski, R. T. (2021). Efeitos da temperatura e do teor de água na qualidade de grãos de canola durante o armazenamento. Revista de Ciência e Inovação, 6 (1), 57-67.

Oliveira, E. R., Queiroz, B. B., Peixoto, C. P., \& Almeida, A. T. (2020). Tempos e formas de armazenamento na qualidade fisiológica de sementes de amendoim. Revista Brasileira de Iniciação Científica, 7 (5), 245-263.

Pereira, R. Z., \& Damaceno, J. B. D. (2018). Tecnologia de secagem de sementes de soja. Desarrollo Local Sostenible, 11 (31) 2-10.

Rodrigues, L. G. S. M., Rodrigues, F. M., Oliveira, E. M., Viera, V. B., Arévalo, A. M., \& Viroli, S. L. M. (2016). Amendoim (Arachis sp.) como fonte na matriz energética brasileira. Journal of Bioenergy and Food Science, 3 (3), 178-190.

Saath, R., Borém, F. M., Alves, E., Taveira, J. H. S., \& Medice, R. (2010). Microscopia eletrônica de varredura do endosperma de café (Coffea arabica L.) durante o processo de secagem. Ciência e Agrotecnologia, 34 (1), 196-203.

Saath, R., Wenneck, G. S., Santi, D. C., Rezende, R., \& Araujo, L. L. (2021). Gestão da qualidade na pós-colheita do amendoim como ferramenta à competitividade. Revista em Agronegócio e Meio Ambiente, 14, (1), e007927. https://doi.org/10.17765/2176-9168.2021v14n1e007927.

Sarath, K. L. L., Goneli, A. L. D., Filho, C. P. H., Masetto, T. E., \& Oba, G. C. (2016). Physiological potential of peanut seeds submitted to drying and storage. Journal of Seed Science, 38 (3), 233-240.

Sarto, D. O. C. C. (2019). Condições de armazenamento e conservação do potencial fisiológico de sementes de diferentes cultivares de amendoim. Escola Superior de Agricultura Luiz de Queiroz, Universidade de São Paulo, 110p. 
Research, Society and Development, v. 10, n. 3, e24910313287, 2021

(CC BY 4.0) | ISSN 2525-3409 | DOI: http://dx.doi.org/10.33448/rsd-v10i3.13287

Smaniotto, T. A. S., Resende, O., Sousa, K. A. de, Rodrigues, G. B., Bessa, J. F. V., \& Resende, L. F. L. (2020). Physiological quality of sunflower seeds stored in different packaging. Research, Society and Development, 9 (6), e47963466. https://doi.org/10.33448/rsd-v9i6.3466

Silva, A. O. da., Silva, A. O. da., Gomes, J. A., Oliveira, R. C. de., Silva, D. A. S., \& Viégas, I. J. M. (2021). Grain storage in Family agriculture: main problems and ways of stroage in the notheast paraense region. Research, Society and Development, 10 (1), e.36610111835. https://doi.org/10.33448/rsdv10i1.11835.

Silva Neto, W. A., \& Santos, T. L. (2019). O déficit na capacidade estática de armazenamento nas regiões centro-oeste e sul do Brasil. Revista De Economia E Agronegócio, 17(3), 507-530. https://doi.org/10.25070/rea.v17i3.8358

Ziegler, V., Ferreira, C. D., Tonieto, L., Silva, J. G., Oliveira, M., \& Elias, M. C. (2017). Efeitos da temperatura de armazenamento de grãos de arroz integral de pericarpo pardo, preto e vermelho sobre as propriedades físico-químicas e de pasta. Brazilian Journal of Food Technology, 20 , e2016051. https://doi.org/10.1590/1981-6723.5116

Wenneck, G. S., Saath, R., Santi, D. C., Araújo, L. L., Santos, J. P., Oliveira, G. G. F. (2020). Hygroscopic balance in pumpkin seeds. Revista de Agricultura Neotropical, 7 (2), 17-26. 